\title{
Improving palliative care with deep learning
}

\author{
Anand Avati ${ }^{*}$, Kenneth Jung ${ }^{2}$, Stephanie Harman ${ }^{3}$, Lance Downing ${ }^{2}$, Andrew Ng${ }^{1}$ and Nigam H. Shah ${ }^{2}$
}

\begin{abstract}
Background: Access to palliative care is a key quality metric which most healthcare organizations strive to improve. The primary challenges to increasing palliative care access are a combination of physicians over-estimating patient prognoses, and a shortage of palliative staff in general. This, in combination with treatment inertia can result in a mismatch between patient wishes, and their actual care towards the end of life.

Methods: In this work, we address this problem, with Institutional Review Board approval, using machine learning and Electronic Health Record (EHR) data of patients. We train a Deep Neural Network model on the EHR data of patients from previous years, to predict mortality of patients within the next 3-12 month period. This prediction is used as a proxy decision for identifying patients who could benefit from palliative care.

Results: The EHR data of all admitted patients are evaluated every night by this algorithm, and the palliative care team is automatically notified of the list of patients with a positive prediction. In addition, we present a novel technique for decision interpretation, using which we provide explanations for the model's predictions.

Conclusion: The automatic screening and notification saves the palliative care team the burden of time consuming chart reviews of all patients, and allows them to take a proactive approach in reaching out to such patients rather then relying on referrals from the treating physicians.
\end{abstract}

Keywords: Deep learning, Palliative care, Electronic health records, Interpretation

\section{Background}

The gap between the desires of patients of how they wish to spend their final days, versus how they actually spend, is well studied and documented. While approximately $80 \%$ of Americans would like to spend their final days at home if possible, only $20 \%$ do [1]. Of all the deaths that happen in the United States, up to $60 \%$ of them happen in an acute care hospital while the patient was receiving aggressive care. Over the past decade access to palliative care resources has been on the rise in the United States. In 2008 , Of all hospitals with fifty or more beds, $53 \%$ of them reported having palliative care teams; which rose to $67 \%$ in 2015 [2]. However, data from the National Palliative Care registry estimates that, despite increasing access, less than half of the $7-8 \%$ of all hospital admissions that need palliative care actually receive it [3]. A major contributor for this gap is the shortage of palliative care workforce [4]. Yet,

*Correspondence: avati@cs.stanford.edu

'Department of Computer Science, Stanford University, Stanford, CA, USA

Full list of author information is available at the end of the article technology can still play a crucial role by efficiently identifying patients who may benefit most from palliative care, but might otherwise slip through the cracks under current care models.

We address two aspects of this problem in our study. First, physicians tend to be overoptimistic, work under extreme time pressures, and as a result may not fail to refer patients to palliative care even when they may benefit [5]. This leads to patients often failing to have their wishes carried out at their end of life [6] and overuse of aggressive care. Second, the shortage of professionals in palliative care makes it expensive and time-consuming for them to proactive identify candidate patients via manual chart review of all admissions.

Another challenge is that the criteria for deciding which patients benefit from palliative care may be impossible to state explicitly and accurately. In our approach, we use deep learning to automatically screen all patients admitted to the hospital, and identify those who are most likely 
to have palliative care needs. Since the criteria for identifying palliative needs could be fuzzy and hard to define precisely, the algorithm addresses a proxy problem - to predict the probability of a given patient passing away within the next 12 months - and use that probability for making recommendations to the palliative care team. This saves the palliative care team from performing manual and cumbersome chart review of every admission, and also helps counter the potential biases of treating physicians by providing an objective recommendation based on the patient's EHR. Currently existing tools to identify such patients have limitations, and they are discussed in the next section.

\section{Related work}

Accurate prognostic information is valuable to patients and caregivers (for setting expectations, planning for care and end of life), and to clinicians (for planning treatment) $[7,8]$. Several studies have shown that clinicians generally tend to be over optimistic in their estimates of the prognoses of terminally ill patients [5, 9-11]. It has also been shown that no subset of clinicians are better at late stage prognostication than others $[12,13]$. However, in practice, the most common method of predictive survival remains to be the clinician's subjective judgment [12]. Several solutions exist that attempt to make patient prognosis more objective and automated. Many of these solutions are models that produce a score based on the patient's clinical and biological parameters, and can be mapped to an expected survival rate.

\section{Prognostic tools in palliative care}

The Palliative Performance Scale [14] was developed as a modification of the Karnofsky Performance Status Scale (KPS) [15] to the Palliative care setting, and is calculated based on observable factors such as: degree of ambulation, ability to do activities, ability to do self-care, food and fluid intake, and state of consciousness. The Palliative Prognostic Score (PPS) was constructed for the Palliative Care setting as well, focusing on terminally ill cancer patients [16]. The PPS is calculated with multiple regression analysis based on the following variables: Clinical Prediction of Survival (CPS), Karnofsky Performance Status (KPS), anorexia, dyspnea, total white blood count (WBC) and lymphocyte percentage. The Palliative Prognostic Index (PPI), developed around the same time as PPS, also calculates a multiple regression analysis based score using Performance Status, oral intake, edema, dyspnea at rest, and delirium. These scores are difficult to implement at scale since they involve face-to-face clinical assessment and involve prediction of survival by the clinician. Furthermore, these scores were designed to be used within the palliative care setting, where the patient is already in an advanced stage of the disease - as opposed to identifying them earlier.

\section{Prognostic tools in the intensive care unit}

There also are prognosis scoring models that are commonly used in the Intensive Care Unit. The APACHE-II (Acute Physiology, Age, Chronic Health Evaluation) Score predicts hospital mortality risk for critically ill hospitalized adults in the ICU [17]. This model has been more recently refined with the APACHE-III Score, which uses factors such as major medical and surgical disease categories, acute physiologic abnormalities, age, preexisting functional limitations, major comorbidities, and treatment location immediately prior to ICU admission [18]. Another commonly used scoring system in the ICU is the Simplified Acute Physiological Score, or SAPS II [19], which is calculated based on the patient's physiological and underlying disease variables. While these score are useful for the treatment team when the patient is already in the ICU, they have limited use in terms of identifying patients who are at risk of longer term mortality, while they are still capable of having a meaningful discussion of their goals and values, so that they can be set on an alternative path of care.

\section{Prognostic tools for early identification}

There have been a number of studies and tools developed that aim to identify terminally ill patients early enough for an end-of-life plan and care to be meaningful.

CriSTAL (Criteria for Screening and Triaging to Appropriate aLternative care) was developed to identify elderly patients nearing end of life, and quantifies the risk of death in the hospital or soon after discharge [20]. CriSTAL provides a check list using eighteen predictors with the goal of identifying the dying patient.

CARING is a tool that was developed to identify patients who could benefit from palliative care [21]. The goal was to use six simple criteria in order to identify patients who were at risk of death within 1 year. PREDICT [22] is a screening tool also based on six prognostic indicators, which were refined from CARING. The model was derived from 976 patients.

The Intermountain Mortality Risk score is an all-causes mortality prediction based on common laboratory tests [23]. The model provides score for 30-day, 1-year and 5 -year mortality risk. It was trained on a population of 71,921 and tested on 47,458 .

Cowen et al. [24] proposed using a twenty-four factor based prediction rule at the time of hospital admission to identify patients with high risk of 30-day mortality, and to organize care activities using this prediction as a context. One of the their motivation was to have a rule from a single set of factors, and not be disease specific. The model was derived from 56,003 patients. 
Meffert et al. [25] proposed a scoring method based on logistic regression on six factors to identify hospitalized patients in need of palliative care. In this prospective study, they asked the treating physician at the time of discharge whether the patient had palliative care needs. The trained model was then used to identify such patients at the time of admission. The model was derived from 39,849 patients.

Ramachandran et al. [26] developed a 30-day mortality prediction tool for hospitalized cancer patients. Their model used eight variables that were based on information from the first $24 \mathrm{~h}$ of admission, and laboratory results and vitals. A logistic regression model was developed from these eight variables and used as a scoring function. The model was derived from 3062 patients.

Amarasingham et al. [27] built a tool to screen patients who were admitted with heart failure, and identify those who are at risk of 30-day readmission or death. Their regression model uses a combination of Tabak Morality Score [28], markers of social, behavioral, and utilization activity that could be obtained electronically, ICD-9 CM codes specific to depression and anxiety, billing and administrative data. Though this study was not specifically focused on palliative care, the methodology of using EHR system data is relevant to our work. The model was derived from 1372 patients.

Makar et al. [29] used only Medicare claims data on older population ( $\geq 65$ years) to predict mortality in six months. By limiting their model to use only administrative data, they hypothesized an easier deployment scenario thereby making automated prognostic models more prevalent. The model was derived separately on four cohorts (one per disease type) with 20,000 patients per cohort.

\section{Prognosis in the age of big-data}

The rapid rise and proliferation of EHR systems in healthcare over the past couple of decades, combined with advances in Machine Learning techniques on high dimensional data provides a unique opportunity to make contributions in healthcare, especially in precision medicine and disease prognosis [30, 31]. All the tools described above, and those we reviewed [32-36], have at least one of the following limitations. They were either derived from small data sets (limited to specific studies or cohorts), or used too few variables (intentionally to make the model portable, or avoid overfitting), or the model was too simple to capture the complexities and subtleties of human health, or was limited to certain sub-populations (based on disease type, age etc.) We address these limitations in our work.

\section{Methods}

We hypothesize, as described earlier, that predicting mortality is a reasonable approximation to predicting palliative needs in patients, though palliative care is applicable more broadly beyond just end of life care, including patients still undergoing painful curative treatments (such as bone marrow transplants, etc). Our approach to the problem of mortality prediction is from the point of view of the palliative care team, by being largely agnostic to disease type, disease stage, severity of admission (ICU vs non-ICU), age etc. The scale of data (in terms of number of patients) allows us to take a deep learning model that considers every patient in the EHR (with a sufficiently long history), without limiting our analysis to any specific sub-population or cohort. We frame a proxy problem statement (in place of identifying palliative needs) in a tractable way as follows:

Given a patient and a date, predict the mortality of that patient within 12 months from that date, using EHR data of that patient from the prior year.

This framing lends itself to be treated as a binary classification problem, and we take a supervised learning approach using deep learning to solve it. Other than building a model that performs well on the above problem, we are also separately interested in its performance on a specific patient sub-population: patients who are currently admitted. This is because it is much easier for the palliative care staff to intervene with admitted patients. This problem formulation and modeling has been previously described in [37].

\section{Data source}

STRIDE (Stanford Translational Research Integrated Database Environment) [38] is a clinical data warehouse supporting clinical and translational research at Stanford University. The data is available in the form of a relational database, from which we extract features and creating a training dataset using SQL queries. The snapshot of STRIDE (version 6) used in our work includes the EHR data of approximately 2 million adult and pediatric patients cared for at either the Stanford Hospital or the Lucile Packard Children's hospital between 1990 and 2014.

\section{Constructing a dataset for supervised learning}

In constructing a supervised learning data set, we define the following concepts:

- Positive cases: Patients who have a recorded date of death in the EHR are considered positive cases.

- Negative cases: Patients who do not have a recorded date of death in the EHR are considered negative cases. 
- Prediction date: The point in time, specific to each patient, that divides the patient's health record timeline into virtual future and past events, is considered that patient's prediction date.

Data from each patient's virtual past is used to calculate the probability of their death 3-12 months in the future. Note that we must take care when defining the prediction date to not violate common sense constraints (described below) that could invalidate the labels. We only include patients for whom it is possible to find a prediction date that satisfies these constraints.

\section{Positive cases}

Palliative care is most beneficial when the referral occurs 3-12 months prior to death. The preparatory time required to start palliative care generally makes it too late for patients who pass away within three months. On the other hand, a lead time longer than 12 months is not desirable either, because making accurate predictions over such a long time horizon is difficult, and more importantly, palliative care interventions are a limited resource that are best focused on more immediate needs. The constraints that the prediction date should meet for positive cases are as follows:

- The prediction date must be a recorded date of encounter.

- The prediction date must be at least 3 months prior to date of death (otherwise death is too near).

- The prediction date can be at most 12 months prior to date of death (otherwise death is too far).

- The prediction date must be at least 12 months after the date of first encounter (otherwise the patient lacks sufficient history on which to base a prediction).

- In-patient admissions are preferred over other admission types for the prediction date, as long as they meet the previous constraints (since it is easier to start the palliative care conversation with them).

- The prediction date must be the earliest among the possible candidate dates subject to previous constraints.

\section{Negative cases}

Negative cases (patients without a date of death) are those patients who were alive for at least 12 months from the prediction date. Their prediction date is chosen such that it satisfies the following constraints:

- The prediction date must be a recorded date of encounter.

- The prediction date must be at least 12 months prior to date of last encounter (to avoid ambiguity of death after date of EHR snapshot).
- The prediction date must be at least 12 months after the date of first encounter (otherwise insufficient history).

- In-patient admissions are preferred over other encounter types for the prediction date, as long as they meet the previous constraints (to serve as controls for the admitted positive cases).

- The prediction date must be the latest among the possible candidate dates subject to previous constraints.

\section{Admitted patients}

These are patients whose prediction date chosen based on the above criteria happens to fall within an in-patient admission. Remaining patients (i.e, patients whose prediction date does not fall in range during an in-patient admission) are considered non-admitted. Note that nonadmitted patients could still have other recorded admissions in their history, just their prediction date did not fall in one of those ranges. Further, we observe that patient records generally get updated with the latest diagnostic and physiological data, such as preliminary tests, diagnostics, etc. within the first twenty four hours of admission. Therefore the second day of admission is generally better suited for making a more informed prediction. Based on this rationale, for admitted patients, we re-adjust their prediction date by incrementing it to be the second day of admission.

For both positive and negative cases, we censor all the data after their corresponding prediction date. The KMplot of censor lengths is shown in Fig. 1, highlighting the separation between the two classes at 365 days.

\section{Data description}

The inclusion criteria resulted in selecting a total of 221,284 patients. Table 1 shows the breakdown of these patients based on inclusion and admission. Note that

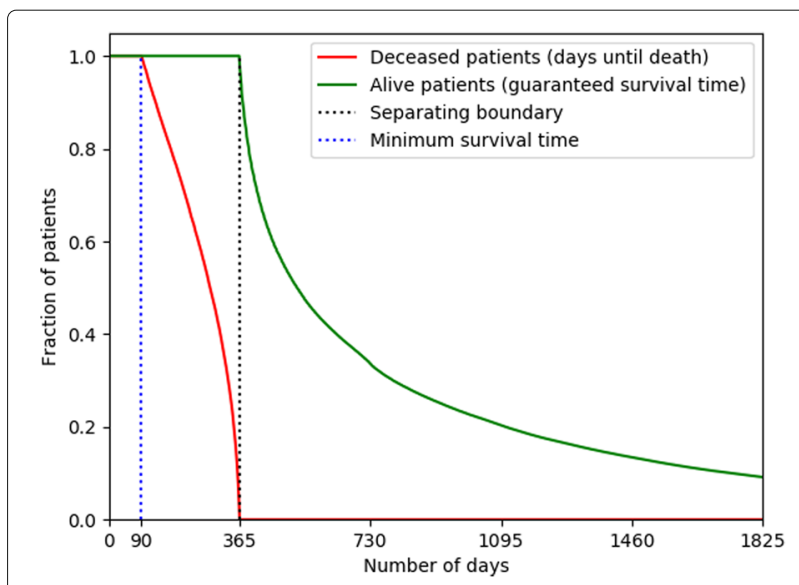

Fig. 1 Right-censoring lengths shown as a survival plot 
Table 1 Breakdown of patient counts

\begin{tabular}{llll}
\hline & Alive & Deceased & Total \\
\hline In EHR & $1,880,096$ & 131,009 & $2,011,105$ \\
Selected & 205,571 & 15,713 & 221,284 \\
Admitted & 9648 & 1131 & 10,779 \\
\hline
\end{tabular}

the admitted patients are kept a subset of the included patients, and not separated into a disjoint set.

We observe that, unsurprisingly, the distribution of age at prediction time is not equal between the classes, and that the positive class (of deceased patients) is skewed towards older age (Fig. 2).

The included patients are randomly split in approximate ratio 8:1:1 into training, validation and test sets, as shown in Table 2.

The prevalence of death among the set of all included patients is approximately $7 \%$. Of all the included patients, approximately $5 \%$ were admitted patients (i.e., those who had their prediction date as the second day of an admission). Among the admitted patients subset, the prevalence of death is a little higher, at about $11 \%$.

\section{Feature extraction}

For each patient, we define their observation window as the 12 months leading up to their prediction date. Within the observation window of each patient, we create features using ICD9 (International Classification of Diseases 9th rev) diagnostic and billing codes, CPT (Current Procedural Terminology) procedure codes, RXCUI prescription codes, and encounters found in that period.

Features are created as follows. We split the observation window of each paitent into four observation slices, specified relative to the prediction date (PD). This is done in order to capture the longitudinal nature of the data.

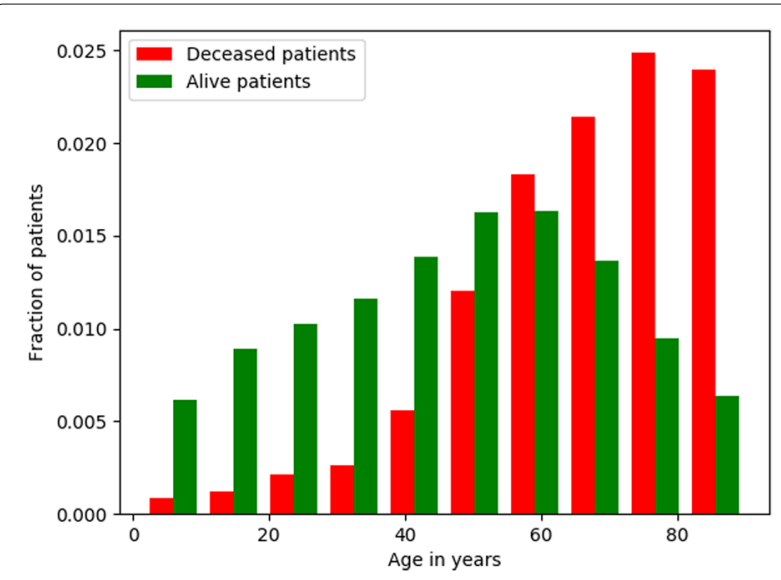

Fig. 2 Age of patients at prediction time
Table 2 Data split for modeling

\begin{tabular}{lllll}
\hline & Training & Validation & Testing & \\
\hline Alive & 164,424 & 20,619 & 20,528 & 205,571 \\
Deceased & 12,587 & 1520 & 1606 & 15,713 \\
& 177,011 & 22,139 & 22,134 & 221,284 \\
\hline
\end{tabular}

The exact split points of each slice within the observation window is shown in Table 3.

Thus, observation slice 1 is the most recent, and observation slice 4 is the oldest. In order to give more emphasis to recent data, the slice widths are intentionally narrow in the later slices compared to the earlier ones. Within each observation slice, we count the the number of occurrences of each code in each code category (prescription, billing, etc.) in that range. The count of every such code within the slice is considered a separate feature.

We also include the patient demographics (age, gender, race and ethnicity), and the following per-patient summary statistics in the observation window for each code category:

- Count of unique codes in the category.

- Count of total number of codes in the category.

- Maximum number of codes assigned in any day.

- Minimum number of codes (non-zero) assigned in any day.

- Range of number of codes assigned in a day.

- Mean of number of codes assigned in a day.

- Variance in number of codes assigned in a day.

All these features (i.e, code counts in each of the four observation slices, per category summary statistics over the observation window, and demographics) were concatenated to form the candidate feature set. From this set, we pruned away those features which occur in 100 or fewer patients. This resulted in the final set of 13,654 features. Of the 13,654 features, each patient on average has 74 non-zero values (with a standard deviation of 62), and up to a maximum of 892 values. The overall feature matrix is approximately $99.5 \%$ sparse.

Table 3 Observation window and slices

\begin{tabular}{llll}
\hline & Start date & End date & Duration \\
\hline Observation window & PD -365 & PD & 365 \\
Observation slice 1 & PD -30 & PD & 30 \\
Observation slice 2 & PD - 90 & PD - 30 & 60 \\
Observation slice 3 & PD - 180 & PD - 90 & 90 \\
Observation slice 4 & PD - 365 & PD - 180 & 185
\end{tabular}




\section{Algorithm and training}

Our model is a Fully Connected Deep Neural Network (DNN) [39] having an input layer (of 13,654 dimensions), 18 hidden layers (of 512 dimensions each) and a scalar output layer. We employ the logistic function and log loss at the output layer for binary classification (with 0/1 labels), and use the Scaled Exponential Linear Unit (SeLU) activation function [40] at each layer. The model is optimized using the Adam optimizer [41], with a mini-batch size of 128 examples. The default learning rate was used (0.001).

Intermediate model snapshots of the model weights were taken every 250 mini-batch iterations, and the snapshot that performed best on the validation test was retroactively selected as the final model. Explicit regularization was not found necessary. The network configuration was reached by extensive hyperparameter search over various network depths (ranging from 2 to 32) and activation functions (tanh, ReLU and SeLU).

The software was implemented using the Python programming language (version 2.7), PyTorch framework [42], and the scikit-learn library (version 0.17.1) [43]. The training was performed on an NVIDIA TitanX (12 GB RAM) with CUDA version 8.0.

\section{Evaluation metric}

Since the data is imbalanced (with $7 \%$ prevalence), accuracy can be a poor evaluation metric [44]. As an extreme case, blindly predicting the majority class without even looking at the data can result in high accuracy, though as useless such a classifier may be. The ROC curve plots the trade-off between sensitivity and specificity, and the Area Under its Curve (AUROC) is generally a more robust metric compared to accuracy in imbalanced problems, but it can also be sometimes misleading $[45,46]$. In use cases where the algorithm is used to surface examples of interest based on a query from a pool of data (e.g "find me patients who are near death") and take action on them, the tradeoff between precision and recall is more meaningful than the tradeoff between sensitivity and specificity. This is generally because the action has a cost associated with it, and precision (or PPV) informs us of how likely that cost results in utility. Therefore, we use the Average Precision (AP) score, also known as Area Under Precision-Recall Curve (AUPRC) for model selection [47].

\section{Results}

In this section we report technical evaluation results obtained on the test set using the model selected based on the best AP score on the validation set.

We observe that the model is reasonably calibrated (Fig. 3) with a Brier score of $\mathbf{0 . 0 4 2}$. In the high threshold regime, which is of interest to us, the model is a little conservative (under-confident) in its probability estimates.

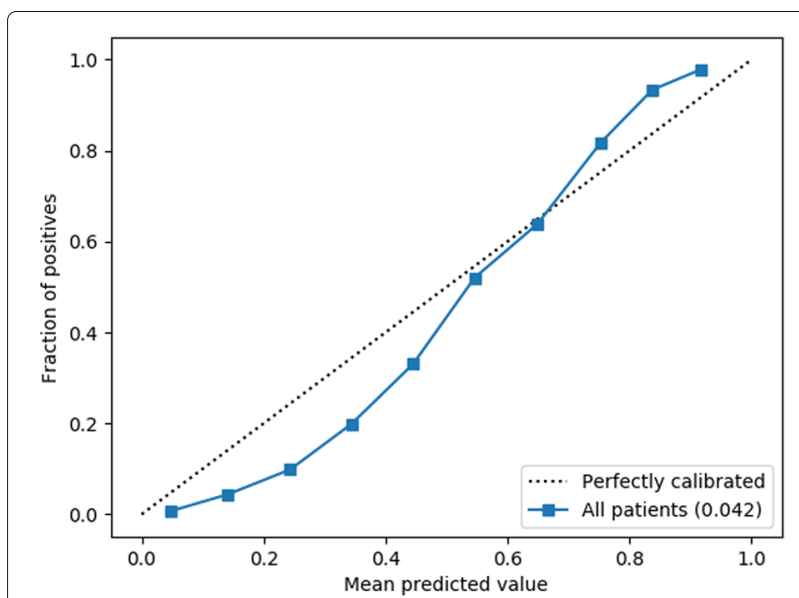

Fig. 3 Reliability curve (calibration plot) of the model output probabilities on the test set data

This means, on average we expect the real precision in the selected candidates to be higher than expected.

The interpolated Precision-Recall curve (with the interpolation performed as explained in Chap 8 of [48]) is shown in Fig. 4. The model achieves an AP score of 0.69 (0.65 on admitted patients). Early recall is desirable, and therefore Recall at precision 0.9 is a metric of interest. The model achieves recall of 0.34 at 0.9 precision $(0.32$ on admitted patients). The Receiver Operating Characteristic curve is shown in Fig. 5. The model achieves an AUROC of 0.93 ( 0.87 for admitted patients). Both the ROC and Precision-Recall plots suggest that the model demonstrates strong early recall behavior.

\section{Qualitative analysis}

It is worth recalling that predicting mortality was only a proxy problem for the original problem of identifying patients who could benefit from palliative care. In






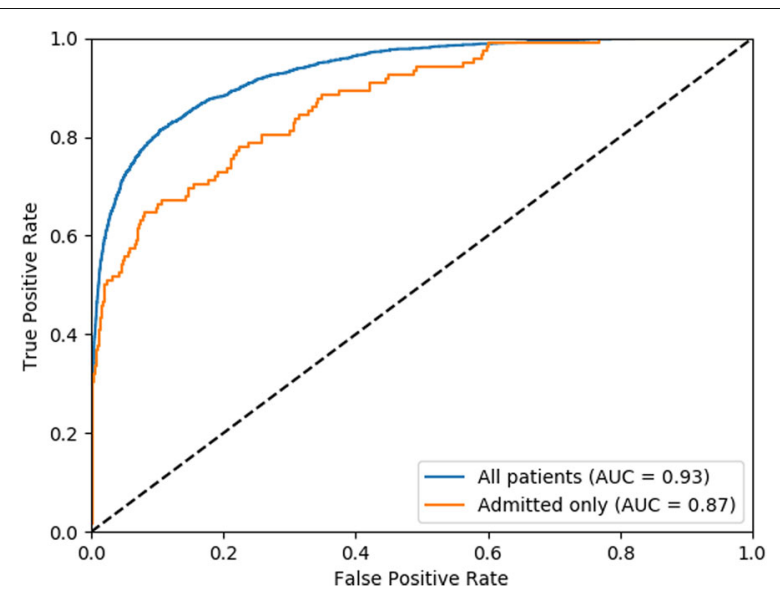

Fig. 5 Receiver Operating Characteristic (ROC) of the model performance on the test set

order to evaluate our performance on the original problem, we inspected false positives with high probability scores. Although such patients did not pass away within 12 months from their prediction dates, we noted that they were often diagnosed with terminal illness and/or are high utilizers of healthcare services. This can be seen in the positive and false positive examples shown in "Discussion" section.

Upon conducting a chart review of 50 randomly chosen patients in the top 0.9 precision bracket of the test set, the palliative care team found all were appropriate for a referral on their prediction date, even if they survived more than a year. This suggests that mortality prediction was a reasonable (and tractable) choice of a proxy problem to solve.

\section{Discussion}

Supervised machine learning techniques, and in particular Deep Learning techniques, have recently demonstrated tremendous success in predictive ability. However, better performance often requires larger, more complex models, which comes with an inevitable cost of sacrificing interpretability. It is worth drawing a distinction between interpreting a model itself, versus interpreting the predictions from the model $[49,50]$. While interpreting complex models (e.g very deep neural networks) may sometimes be infeasible, it is often the case that users only want an explanation for the prediction made by the model for a given example. The utility of such an interpretation is generally a function of the nature of action taken based on the prediction. If the action is a highstake action, such as an automated clinical decision, it is important to establish the trust of the practitioner in the model's decisions for them to feel comfortable with the actions, and providing explanations along with decisions help establish that trust. In the context of our work, the action is not an automated clinical decision, but rather a tool to make the workflow of a human more efficient

Table 4 Prediction explanation generated on a random positive patient with high probability score

\begin{tabular}{lllll}
\hline Patient MRN & XXXXXXX & & & \\
Probability score & 0.946 & & & \\
Factors & Code & Value & Influence & Description \\
\hline Top Diagnostic factors & V10.51 & 4 & 0.0051 & Personal history of malignant neoplasm of bladder \\
& V10.46 & 5 & 0.0019 & Personal history of malignant neoplasm of prostate \\
& 518.5 & 1 & 0.0012 & Pulmonary insufficiency following trauma and surgery \\
& 518.82 & 1 & 0.0008 & Other pulmonary insufficiency \\
Top Procedural factors & 88.75 & 1 & 0.0006 & Diagnostic ultrasound of urinary system \\
& 75331 & 1 & 0.0017 & Pathology consultation during surgery with FS \\
& 72158 & 1 & 0.0014 & Transcatheter Diagnostic Radiology Procedure \\
& Code_Type_Count & 76 & 0.0013 & MRI and CT Scans of the Spine \\
Top Medication factors & 76005 & 1 & 0.0007 & Fummary statistic (count of all ICD/CPT codes) \\
Top Encounter factors & Hx Scan & & & \\
& Inpatient & 21 & 0.0012 & Number of scan encounters of all types \\
& Var_Codes_per_Day & 8 & 0.0002 & Summary statistic (variance in number of codes assigned per day) \\
& Code_Day_Count & 88 & 0.0001 & Number of days any encounter code was assigned \\
Top Demographic factors & Age & 81 & 0.0010 & Age of patient in years at prediction time
\end{tabular}


(i.e avoid cumbersome chart reviews), and the human (i.e palliative care doctor) is always in the loop to make the decision of whether or not to initiate a consult after having a closer look at the patient's history. In such cases, the utility of interpretations is to make the human feel that it's worth their time to even go by the recommendations made by the model. Decision interpretation can also help identify when non-stationarity of the patient data is reaching a certain threshold. We can get an early feel for whether the patient population over time has started drifting away from the data distribution the model was trained on, when the interpretations no longer meet common sense expectations. This could suggest it may be time to either re-tune or retrain the model with more recent data.

We make the following observations to motivate our explanation technique.

- We can view the EHR data as a strictly growing log of events, and that new data is only added (nothing is modified or removed in general). This results in all our features being positive valued (as counts, means and variance of counts, etc).
- We are most interested in explaining why a model assigns high probability to a patient. We are less interested in getting an explanation for why a healthy person was given a low probability (the reasons are also much less clear: the patient did not have brain cancer, did not have pneumonia, and so on).

- Directly perturbing feature vectors (e.g sensitivity analysis or for techniques described in [49]) does not work well in our case. For example, perturbing the feature representing the ICD count for brain cancer from zero to non zero can increase the probability of death significantly, implying that it is an important factor in general. However, that is not a very useful observation for a specific patient who does not have brain cancer.

These observations motivate the following technique. For each ICD-9, CPT, RXCUI and Encounter type, we ablate all occurrences of that code from the patient's EHR, create a new feature vector, and measure the drop in log-probability compared to the original probability. This corresponds to asking the counter-factual: all else being equal, how would the probability change if this patient

Table 5 Prediction explanation generated on a random false positive patient with high probability score

\begin{tabular}{|c|c|c|c|c|}
\hline Patient MRN & YYYYYYY & & & \\
\hline Probability score & 0.909 & & & \\
\hline Factors & Code & Value & Influence & Description \\
\hline \multirow{3}{*}{ Top Diagnostic factors } & 154.1 & 3 & 0.1254 & Malignant neoplasm of rectum \\
\hline & 287.5 & 1 & 0.0194 & Thrombocytopenia, unspecified \\
\hline & 733.90 & 1 & 0.0113 & Other and unspecified disorders of bone and cartilage \\
\hline \multirow[t]{4}{*}{ Top Procedural factors } & 73560 & 1 & 0.0502 & Diagnostic Radiology (Diagnostic Imaging) Procedures of the Lower Extremities \\
\hline & Code_Type_Count & 20 & 0.0491 & Summary statistic (Number of unique ICD-9/CPT codes) \\
\hline & 74160 & 1 & 0.0381 & Diagnostic Radiology (Diagnostic Imaging) Procedures of the Abdomen \\
\hline & Max_Codes_per_Day & 6 & 0.0234 & Summary statistic (Maximum number of codes in any day) \\
\hline \multirow{3}{*}{ Top Medication factors } & Range_Codes_per_Day & 5 & 0.0023 & Summary statistic (Ranges of codes across days) \\
\hline & Max_Codes_per_Day & 5 & 0.0023 & Summary statistic (Maximum number of codes in any day) \\
\hline & Code_Type_Count & 6 & 0.0015 & Summary statistic (Number of unique medication codes) \\
\hline \multirow[t]{4}{*}{ Top Encounter factors } & $\mathrm{H} \times \mathrm{Scan}$ & 19 & 0.2239 & Number of scan encounters of all types \\
\hline & Code_Day_Count & 97 & 0.0284 & Number of days any encounter code was assigned \\
\hline & Outpatient & 22 & 0.0228 & Number of Outpatient encounters \\
\hline & Var_Codes_per_Day & 1 & 0.0074 & Summary statistic (variance in number of codes assigned per day) \\
\hline
\end{tabular}


was not diagnosed with $\mathrm{XYZ}$, prescribed drug $\mathrm{ABC}$, etc? This drop in log-probability is considered the influence the code has on the model's decision for that patient. Demographic features are handled as follows. We zero out the age and swap the gender to the opposite sex, and measure the respective drops in probability. Finally we sort the codes in descending order by influence, and pick the top 5 in each code category. A random example of such a positive and false positive case are shown in Tables 4 and 5 .

\section{Conclusion}

We demonstrate that routinely collected EHR data can be used to create a system that prioritizes patients for follow up for palliative care. In our preliminary analysis we find that it is possible to create a model for all-cause mortality prediction and use that outcome as a proxy for the need of a palliative care consultation. The resulting model is currently being piloted for daily, proactive outreach to newly admitted patients. We will collect objective outcome data (such as rates of palliative care consults, and rates of goals of care documentation) resulting from the use of our model. We also demonstrate a novel method of generating explanations from complex deep learning models that helps build confidence of practitioners to act on the recommendations of the system.

\section{Acknowledgments}

We thank the Stanford Research IT team for their support and help in this project. Research IT, and the Stanford Clinical Data Warehouse (CDW) are supported by the National Center for Research Resources and the National Center for Advancing Translational Sciences, National Institutes of Health, through grant UL1 TR001085. The content of studies done using the CDW is solely the responsibility of the authors and does not necessarily represent the official views of the NIH. We thank Ziang Xie, and the anonymous reviewers for their helpful and insightful comments.

\section{Funding}

Publication costs were funded by grant GCDDG.

\section{Availability of data and materials}

Data used for this study is from Stanford University Clinical Data Warehouse, hosted by the Stanford Research IT.

\section{About this supplement \\ This article has been published as part of BMC Medical Informatics and Decision Making Volume 18 Supplement 4, 2018: Selected articles from the IEEE BIBM International Conference on Bioinformatics \& Biomedicine (BIBM) 2017: medical informatics and decision making. The full contents of the supplement are available online at https://bmcmedinformdecismak.biomedcentral.com/ articles/supplements/volume-18-supplement-4.}

\section{Authors' contributions}

$\mathrm{SH}$ and NHS envisaged the project. AA and $\mathrm{KJ}$ built the model and implemented code, guided by LD, AN and NHS. All authors contributed to the writing of the manuscript. All authors have read and approved final manuscript.

\section{Ethics approval and consent to participate}

This work has obtained ethics approval by the Institutional Review Board of Stanford University under protocol 42078.

\section{Consent for publication}

Not applicable.
Competing interests

The authors declare that they have no competing interests.

\section{Publisher's Note}

Springer Nature remains neutral with regard to jurisdictional claims in published maps and institutional affiliations.

\author{
Author details \\ ${ }^{1}$ Department of Computer Science, Stanford University, Stanford, CA, USA. \\ ${ }^{2}$ Center for Biomedical Informatics Research, Stanford University, Stanford, CA, \\ USA. ${ }^{3}$ Department of Medicine, Stanford University School of Medicine, \\ Stanford, CA, USA.
}

Published: 12 December 2018

\section{References}

1. Where do americans die? Available: https://palliative.stanford.edu/homehospice-home-care-of-the-dying-patient/where-do-americans-die/. Accessed 15 Apr 2017.

2. Dumanovsky T, Augustin R, Rogers M, Lettang K, Meier DE, Sean Morrison R. Special Report The Growth of Palliative Care in U.S. Hospitals: A Status Report. J Palliat Med. 2016;19(1):8-15.

3. palliative care, report card. 2015. Available: https://web.archive.org/web/ 20170316014732/https://reportcard.capc.org/\#key-findings. Accessed 15 Apr 2017

4. Spetz J, Dudley N, Trupin L, Rogers M, Meier DE, Dumanovsky T. Few Hospital Palliative Care Programs Meet National Staffing

Recommendations. Health Aff (Project Hope). 2016;9(9):1690-7.

5. Christakis N, Lamont E. Extent and determinants of error in doctors prognoses in terminally ill patients: prospective cohort study. BMJ Clin Res. 2000;320(7233):469-72.

6. most physicians would forgo aggressive treatment for themselves at the end of life, study finds. 2014. Available: https://med.stanford.edu/news/ all-news/2014/05/most-physicians-would-forgo-aggressive-treatmentfor-themselves-.html. Accessed 15 Apr 2017.

7. Kutner JS, Steiner JF, Corbett KK, Jahnigen DW, Barton PL. Information needs in terminal illness. Soc Sci Med. 1999;48(10):1341-52.

8. Steinhauser KE, Christakis NA, Clipp EC, Mcneilly M, Grambow S, Parker J, Tulsky JA. Preparing for the End of Life: Preferences of Patients, Families, Physicians, and Other Care Providers. J Pain Symptom Manag J Pain Symptom Manage. 2001;2222:727.

9. Selby D, Chakraborty A, Lilien T, Stacey E, Zhang L, Myers J. Clinician Accuracy When Estimating Survival Duration: The Role of the Patient's Performance Status and Time-Based Prognostic Categories. J Pain Symptom Manag. 2011;42(4):578-88.

10. Viganó A, Dorgan M, Bruera E, Suarez-Almazor ME. The Relative Accuracy of the Clinical Estimation of the Duration of Life for Patients with End of Life Cancer. Cancer. 1999;86(1):170-6.

11. Glare $P$, Sinclair $C$, Downing $M$, Stone $P$, Maltoni $M$, Vigano A. Predicting survival in patients with advanced disease. 2008;4:2.

12. White N, Reid F, Harris A, Harries P, Stone P, Pulenzas N. A Systematic Review of Predictions of Survival in Palliative Care: How Accurate Are Clinicians and Who Are the Experts? PLoS ONE. 2016;11 (8):e0161407.

13. Macmillan DO. Accuracy of prediction of survival by different professional groups in a hospice. Palliat Med. 1998;12:117-8.

14. Lau F, Downing GM, Lesperance M, Shaw J, Kuziemsky C. Use of Palliative Performance Scale in End-of-Life Prognostication. J Palliat Med. 2006;9(5):1066-75.

15. Karnofsky DA, Abelmann WH, Craver LF, Burchenal JH. The use of the nitrogen mustards in the palliative treatment of carcinoma. With particular reference to bronchogenic carcinoma. Cancer. 1948;1 (4):634-56.

16. Pirovano M, Maltoni $M$, Nanni O, Marinari M, Indelli M, Zaninetta $G$, Petrella V, Barni S, Zecca E, Scarpi E, Labianca R, Amadori D, Luporini G. A New Palliative Prognostic Score: A First Step for the Staging of Terminally III Cancer Patients for the Italian Multicenter and Study Group on Palliative Care. J Pain Symptom Manag Pirovano et al. 1997;17(4):231-9.

17. Knaus WA, Draper EA, Wagner DP, Zimmerman JE. APACHE II: a severity of disease classification system. Crit Care Med. 1985;13(10):818-29.

18. Knaus WA, Wagner DP, Draper EA, Zimmerman JE, Bergner M, Bastos PG, Sirio CA, Murphy DJ, Lotring T, Damiano A, Harrell FE. The APACHE III Prognostic System. Chest. 1991;100(6):1619-36. 
19. Le Gall J-R, Lemeshow S, Saulnier F. A New Simplified Acute Physiology Score (SAPS II) Based on a European/North American Multicenter Study. J Am Med Assoc. 1993;12(24):2957.

20. Cardona-Morrell M, Hillman K. Development of a tool for defining and identifying the dying patient in hospital: Criteria for Screening and Triaging to Appropriate aLternative care (CriSTAL). BMJ Support Palliat Care. 2015;3(1):78-90.

21. Fischer SM, Gozansky WS, Sauaia A, Min S-J, Kutner JS, Kramer A. A Practical Tool to Identify Patients Who May Benefit from a Palliative Approach: The CARING Criteria. J Pain Symptom Manag. 2006;31(4): 285-92.

22. Richardson P, Greenslade J, Shanmugathasan S, Doucet K, Widdicombe N, Chu K, Brown A. PREDICT: a diagnostic accuracy study of a tool for predicting mortality within one year: Who should have an advance healthcare directive? Palliat Med. 2015;29(1):31-7.

23. Horne BD, May HT, Muhlestein JB, Ronnow BS, Lappè DL, Renlund DG, Kfoury AG, Carlquist JF, Fisher PW, Pearson RR, Bair TL, Anderson JL. Exceptional Mortality Prediction by Risk Scores from Common Laboratory Tests. AJM. 2009;122:550-8.

24. Cowen ME, Strawderman RL, Czerwinski JL, Smith MJ, Halasyamani LK, Cowen ME. Mortality predictions on admission as a context for organizing care activities. J Hosp Med. 2013;5(5):229-35.

25. Meffert C, Rücker G, Hatami I, Becker G. Identification of hospital patients in need of palliative care-a predictive score. BMC Palliat Care. 2016;15. https://doi.org/10.1186/s12904-016-0094-7.

26. Ramchandran KJ, Shega JW, Von Roenn J, Schumacher M, Szmuilowicz E, Rademaker A, Weitner BB, Loftus PD, Chu IM, Weitzman S. A predictive model to identify hospitalized cancer patients at risk for 30-day mortality based on admission criteria via the electronic medical record. Cancer. 2013;6(11):2074-80.

27. Amarasingham R, Moore BJ, Tabak YP, Drazner MH, Clark CA, Zhang S, Reed WG, Swanson TS, Ma Y, Halm EA. An Automated Model to Identify Heart Failure Patients at Risk for 30-Day Readmission or Death Using Electronic Medical Record Data. Med Care. 2010;48(11):981-8.

28. Tabak YP, Johannes RS, Silber JH. Using Automated Clinical Data for Risk Adjustment. Medical Care. 2007;45(8):789-805.

29. Makar M, Ghassemi M, Cutler D, Obermeyer Z. Short-Term Mortality Prediction for Elderly Patients Using Medicare Claims Data. Int J Mach Learn Comput. 2015;5(3):192-7.

30. Sim I, FH Z, SMT, SY H, N Z, Y H, M N. Two Ways of Knowing: Big Data and Evidence-Based Medicine. Ann Intern Med. 2016;164(8):562.

31. Obermeyer Z, Emanuel EJ. Predicting the Future - Big Data, Machine Learning, and Clinical Medicine. N Engl J Med. 2016;9(13):1216-9.

32. Rose S. Mortality Risk Score Prediction in an Elderly Population Using Machine Learning. Am J Epidemiol. 2013;3(5):443-52.

33. Wiemken TL, Furmanek SP, Mattingly WA, Guinn BE, Cavallazzi R, Fernandez-Botran R, Wolf LA, English CL, Ramirez JA. Predicting 30-day mortality in hospitalized patients with community-acquired pneumonia using statistical and machine learning approaches. J Respir Infect. 2017;1(3):5.

34. Motwani M, Dey D, Berman DS, Germano G, Achenbach S, Al-Mallah MH, Andreini D, Budoff MJ, Cademartiri F, Callister TQ, Chang H-J, Chinnaiyan K, Chow BJ, Cury RC, Delago A, Gomez M, Gransar H, Hadamitzky M, Hausleiter J, Hindoyan N, Feuchtner G, Kaufmann PA, Kim Y-J, Leipsic J, Lin FY, Maffei E, Marques H, Pontone G, Raff G, Rubinshtein R, Shaw LJ, Stehli J, Villines TC, Dunning A, Min JK, Slomka PJ. Machine learning for prediction of all-cause mortality in patients with suspected coronary artery disease: a 5-year multicentre prospective registry analysis. Eur Heart J. 2016;25(7):ehw188.

35. Cooper GF, Aliferis CF, Ambrosino R, Aronis J, Buchanon BG. An Evaluation of Machine-Learning Methods for Predicting Pneumonia Mortality. Artif Intell Med. 1997;9(2):107-38.

36. Vomlel J, Kružík H, Tma P, Přeček J, Hutyra M. Machine Learning Methods for Mortality Prediction in Patients with ST Elevation Myocardial Infarction. In the Proceedings of The Nineth Workshop on Uncertainty Processing WUPES'12, Mariánské Lázně, Czech Republic. 2012. p. 204-213.

37. Avati A, Jung K, Harman S, Downing L, Ng A, Shah NH. Improving palliative care with deep learning. IEEE; 2017. p. 311-316. Available: http:// ieeexplore.ieee.org/document/8217669/.

38. Lowe HJ, Ferris TA, Hernandez Nd PM, Weber SC. STRIDE - An Integrated Standards-Based Translational Research Informatics Platform. In: AMIA
Annual Symposium Proceedings. 2009. p. 391-5. Conference paper (AMIA Symposium).

39. LeCun Y, Bengio Y, Hinton G. Deep Learning. Nature. 2015;521:436-44.

40. Klambauer G, Unterthiner T, Mayr A, Hochreiter S Guyon I, Luxburg UV, Bengio S, Wallach H, Fergus R, Vishwanathan S, Garnett R, (eds). Self-normalizing neural networks; 2017. p. 972-981. Curran Associates, Inc. Available: http://papers.nips.cc/paper/6698-self-normalizing-neuralnetworks.pdf. Accessed 2 Nov 2018.

41. Kingma DP, Ba J. Adam: A Method for Stochastic Optimization. In: Proceedings of the 3rd International Conference on Learning Representations (ICLR). 2014. Conference paper (ICLR).

42. Pytorch. Available: https://pytorch.org. Accessed 2 Nov 2018.

43. Pedregosa F, Varoquaux G, Gramfort A, Michel V, Thirion B, Grisel O, Blondel M, Prettenhofer P, Weiss R, Dubourg V, Vanderplas J, Passos A, Cournapeau D, Brucher M, Perrot M, Duchesnay E. Scikit-learn: Machine learning in Python. J Mach Learn Res. 2011;12:2825-30.

44. He H, Garcia E. Learning from Imbalanced Data. IEEE Trans Knowl Data Eng. 2009;9(9):1263-84.

45. Davis J, Goadrich M. The Relationship Between Precision-Recall and ROC Curves. In: Proceedings of the 23rd international conference on Machine learning. 2006. p. 233-40. Conference paper (ICML).

46. Boyd K, Santos Costa V, Davis J, Page CD. Unachievable Region in Precision-Recall Space and Its Effect on Empirical Evaluation. In: Proceedings of the 29th International Coference on International Conference on Machine Learning Pages. 2012. p. 1619-26. Conference paper (ICML).

47. Yuan $Y$, Su W, Zhu M. Threshold-free measures for assessing the performance of medical screening tests. Front Public Health. 2015;3:57.

48. Manning CD, Raghavan $P$, Schütze H. Introduction to Information Retrieval. New York: Cambridge University Press; 2008. Conference paper (KDD).

49. Ribeiro MT, Singh S, Guestrin C. Why Should I Trust You?: Explaining the Predictions of Any Classifier. In: Proceedings of the 22Nd ACM SIGKDD International Conference on Knowledge Discovery and Data Mining. 2016. p. 1135-44.

50. Lipton ZC. The Mythos of Model Interpretability. Queue. 2018;16(3). https://doi.org/10.1145/3236386.3241340.
Ready to submit your research? Choose BMC and benefit from:

- fast, convenient online submission

- thorough peer review by experienced researchers in your field

- rapid publication on acceptance

- support for research data, including large and complex data types

- gold Open Access which fosters wider collaboration and increased citations

- maximum visibility for your research: over $100 \mathrm{M}$ website views per year

At BMC, research is always in progress.

Learn more biomedcentral.com/submissions 